\title{
Marcas técnico-formales de la polémica literaria en la Escuela Poética Siciliana ${ }^{1}$
}

\author{
Ana Ma DOMÍNGUEZ FERRO \\ Departamento de Filología Francesa e Italiana \\ Área de Filología Italiana \\ Universidad de Santiago de Compostela \\ anamaria.dominguez@usc.es
}

\begin{abstract}
RESUMEN
El trabajo pretende abordar el análisis de las composiciones de la Escuela poética siciliana del s. XIII, pertenecientes al género dialógico de la tenzone, en las que se establece un debate en torno a cuestiones relativas a la teorización amorosa. Dentro de este corpus textual, el artículo aborda las marcas retórico-estilísticas empleadas para desarrollar la argumentación y las fórmulas expresivas que los poetas utilizan para entablar la discusión y la polémica.
\end{abstract}

Palabras clave: Lírica italiana del siglo XIII, polémica literaria, Giacomo da Lentini, Abate di Tivoli, Iacopo Mostacci, Piero della Vigna.

[Recibido, septiembre 2011; aprobado, diciembre 2011]

Formal technical signs of the literary polemic

in the Sicilian poetic school

\begin{abstract}
This paper tries to approach the analysis of the composition of the XIII century Sicilian poetic school, those of the dialogic gender of the tenzone, in which there appears a debate about elements relative to the theory of love. In this textual corpus the paper studies the stylistic rhetorical signs used to develop the argumentation and the expressive formulae that poets use to engage, the so-called debate and discussion.
\end{abstract}

Keywords: Italian poetry of the XIII century, literary polemic, Giacomo da Lentini, Abate di Tivoli, Iacopo Mostacci, Piero della Vigna

\footnotetext{
${ }^{1}$ Este trabajo deriva del Proyecto de Investigación El sirventés literario en la lírica románica medieval (FFI2008-05481) coordinado por la Prof. Mercedes Brea, de la Universidad de Santiago de Compostela, y financiado por el MICINN.
} 


\section{Introducción}

El campo de estudio del siguiente trabajo comprende las composiciones poéticas de la escuela siciliana, pertenecientes al género dialógico de la tenzone, en las que se desarrolla un debate o una polémica en torno a cuestiones de carácter literario, en el ámbito de la teorización amorosa. Dado que el tema es general y no aborda aspectos particulares ni personales, sino más bien asuntos relacionados con el arte de trobar o cuestiones relativas a teoría amorosa, en él se ven involucrados, de alguna manera, no sólo los poetas que intercambian sus versos sino todos los miembros de una comunidad o de un círculo intelectual interesados en el mismo tema. Por ello, no es necesaria la alusión concreta a un interlocutor ni la referencia directa para que surja la respuesta y se establezca el diálogo aunque, como veremos, en la mayor parte de las ocasiones hay interconexiones métrico-estilísticas y alusiones internas que remiten a un autor concreto, por lo que se sobreentiende que ha existido una discusión o debate previo con otro u otros colegas.

Dentro de este corpus textual el análisis se centrará en las marcas retóricas utilizadas para introducir el debate literario, los recursos más habituales para establecer la argumentación o la contraargumentación, y las fórmulas expresivas que los poetas ponen al servicio de la discusión o la polémica.

En el contexto de la lírica provenzal, si la canso es el soporte más adecuado del que disponen los trovadores para la poesía amorosa, la ira, la reprensión, el ataque virulento, la polémica literaria y el discurso moralizador encuentran su medio de expresión en el género llamado sirventés ${ }^{2}$. Dos rasgos fundamentales alejan el género utilizado por los poetas italianos de sus predecesores occitanos: En primer lugar, un componente formal, dado que el sirventés se sirve del estrofismo y la melodía de una preexistente canción y, por lo tanto, nos encontramos ante composiciones estructuralmente derivativas. La tenzone italiana en cambio, se presenta como un piccolo macrotesto (Picone 1979: 19) en el que el metro y la rima no vienen predeterminados por una canso anterior, y la autonomía y libertad de cada autor se manifiestan mayoritariamente a través de la forma estrófica del soneto.

Por otra parte, desde el punto de vista temático, si bien los temas son hetereogéneos, parece connatural al género del sirventés el enfoque satírico y el vituperio ${ }^{3}$. Es cierto, como veremos más adelante, que algunos de los textos

\footnotetext{
${ }^{2}$ Las Leys d'Amors lo definen del siguiente modo: "Sirventés es dictatz ques servish al may de vers o de chanso en doas cauzas. la una. cant al compas de las coblas. lautra cant al so. e deu hom entendre: cant al compas. sos assaber que tenga lo compas solamen. ses las acordansas. oz am las acordansas. daquelas meteyshas dictios. o dautras semblans ad aquelas per acordansa. e deu tractar de reprehensio. o de maldig general. per castiar los fols e los malvatz. o pot tractar quis vol. del fag dalquna guerra". Cfr. G. Molinier, Las Flors del Gay Saber estier dichas Las Leys d'Amors, Genève, Slatkine, 1077, II, p. 340.

${ }^{3}$ V. Cian señala: "Uc Faidit ne aveva data una definizione, che comunque esagerata, per noi riesce singularmente preziosa, poiché da essa parrebbe che il sirventese dovesse essere esclusiva-
} 
italianos no están exentos de ironía pero, con carácter general, ésta no es una característica propia de las composiciones que nos proponemos analizar, puesto que el tema no es el ataque personal y directo, sino la discusión metaliteraria en la que las posturas de varios autores pueden entrar en conflicto y en la que la polémica es mucho más sutil. En palabras de Claudio Giunta:

La tenzone nasce non come forma per il dialogo su vicende personali bensì come genere oggettivo, l'unico genere oggettivo-ragionativo che figuri nel repertorio dei siciliani, rappresentando come il rovescio e il complemento teorico della lirica amorosa, lo spazio -l'unico spazio- per la messa a punto di una filosofia dell'amore. (Giunta 2002: 220)

\section{Corpus}

El campo de estudio que hemos delimitado abarca, como anunciábamos al inicio, las tenzoni pertenecientes al corpus siciliano en las que se ven involucrados varios poetas y que tratan un argumento metaliterario, lo que no excluye, como veremos, la invectiva, más o menos velada, o el ataque sutil, entre dos contendientes que defienden puntos de vista diferentes. Dentro del corpus estrictamente siciliano analizaremos las dos tenzoni de Giacomo da Lentini, la primera de ellas con el Abate di $\operatorname{Tivoli}^{4}$ (A) y la segunda con Iacopo Mostacci y Piero della Vigna ${ }^{5}$ (B) así como una tercera anónima $(C)^{6}$.

\subsection{Giacomo da Lentini-Abate di Tivoli}

La tenzone A inaugura el debate duecentesco sobre la fenomenología amorosa $^{7}$. El Abate di Tivoli inicia la composición "Ai deo d'amore a te faccio preghera" (1.18a) con un apóstrofe mediante el cual solicita justicia y, tras la captatio benevolentiae del exordio, declara en los tercetos la herida amorosa que le ha causado el dardo de oro, por lo que pide que otro tanto, a través del dardo de plomo, le suceda a la dama, culpable de su sufrimiento ${ }^{8}$. Este primer soneto se presenta, en principio, como una composición autónoma en la que no parece que se haga referencia a una polémica previa; sin embargo, en el segundo verso (ca mi 'ntendiate s'io chero razone) se sobreentiende un debate con

mente satirico: Sirventes, id est cantio facta vituperio alicuius". Vid. Storia dei generi letterari italiani. La satira, Milano, Vallardi, 1923, vol. I, p. 80.

${ }^{4}$ Cfr. I poeti della Scuola Siciliana, Milano, Mondadori, 2008: vol. Giacomo da Lentini, a cura di R. Antonelli, pp. 349-385 (PSS I) y vol. II Poeti della corte di Federico II dir. C. Di Girolamo (PSS II).

${ }^{5}$ PSS I, pp. 389-411.

${ }_{7}^{6}$ PSS II, pp. 1008-1017.

${ }^{7}$ No me detendré en cuestiones relativas a la temática de la polémica, puesto que es un asunto ya tratado desde el clásico estudio de S. Santangelo, Le tenzoni poetiche nella letteratura italiana delle origini, Genève, Olschki, 1928, hasta otros más actuales como el de C. Giunta, Due saggi sulla tenzone, Roma, Antenore, 2002 o el de S. Marcenaro, "Polemiche letterarie nella lirica italiana del Duecento" Revista de Filologia Románica, 2010, 27, pp. 77-99.

${ }^{8}$ Vid. la discusión textual en PSS I, p. 364 y ss. 
otro u otros colegas. No está claro, de todas formas, que el verso aluda a una situación anterior. Si lo está, en cambio, en el soneto de Giacomo en el que claramente apunta a un destinatario interno, al que no se alude directamente, sino más bien a través de fórmulas generales. El interlocutor, no obstante, es consciente de que la respuesta va dirigida a él, puesto que el Notaro sitúa el término feruto al inicio del incipit, adjetivo que en la composición del Abate aparece en el v. 10 en posición de rima, pero planteando una situación personal y particular: "Feruto sono isvarïatamente" (1.18b), que continúa en el siguiente verso con una interrogación retórica que pretende abrir el debate y desarrollar la cuestión mediante diversas fórmulas. En el tercer verso (Per ch'eo vi saccia dir lo convenente) se dirige a una $2^{\mathrm{a}}$ persona -claramente el Abate- $\mathrm{y}$, en el siguiente, una referencia a quelli che del trovar no ánno posa (v. 4) incluye a todos aquellos que comparten la teoría del Abate.

La respuesta del Abate $(1.18 \mathrm{c})$ se dirige directamente a un interlocutor, a través del apóstrofe amico (v. 3), al que se refiere varias veces a lo largo de la composición, mediante la $2^{\mathrm{a}}$ persona. Giacomo (1.18d) no responde directamente, aunque se defiende de las acusaciones del Abate. El soneto de respuesta de este último (1.18e) que cierra la tenzone contiene referencias directas al Notaro, a lo largo de toda la composición: ser Giacomo valente (v. 2), Lentino (v. 4), 'l più fino (v. 8).

A excepción de la primera composición, que se presenta como texto autónomo, en las demás el Abate di Tivoli dirige sus composiciones de una manera directa a un interlocutor que se revela en el último soneto como Giacomo da Lentini; sin embargo, este último siempre lo hace de modo general, con alusiones internas y referencias más o menos veladas.

\subsection{Giacomo da Lentini-Iacopo Mostacci-Piero della Vigna}

El segundo debate (B) implica a tres poetas que utilizan un tono marcadamente diverso del utilizado en las rimas anteriores. El tema es también la naturaleza del amor pero, a diferencia del caso precedente, en el soneto que inicia la tenzone Iacopo Mostacci (1.19a) plantea una duda con dos posibles soluciones (si el amor es sustancia o accidente): un dubio che mi mise ad averela voi lo mando per determinare (vv. 3-4) aunque él se adhiere a una de ellas (amor es accidente). La respuesta, en este caso, a la quaestio la aportan dos de los poetas de mayor prestigio de la escuela: Piero della Vigna (1.19b) y Giacomo da Lentini (1.19c) ofreciendo cada uno de ellos una solución diferente al problema planteado y entrando en polémica. El esquema métrico de las tres composiciones es idéntico y, aunque no se reproduce el mismo sistema rítmico, las conexiones y referencias internas entre los tres sonetos son muy estrechas. Para M. Picone esta tenzone presenta una configuración textual nueva y original frente a la tradición puesto que aúna varios subgéneros occitánicos como la tenso (discusión sobre un problema amoroso), el partimen (dos soluciones a la cuestión planteada), el tornejamen (tres poetas implicados) y la cobla (tres textos poéticos aislados) (Picone 1979: 19). 
La composición que abre la disputa y el problema planteado está dirigido a un voi (v. 4), que debe despejar la duda. El destinatario vuelve a estar presente en el penúltimo verso, donde se le apremia para que dirima la cuestión ( $m a$ zo che è, da voi voglio audire, v. 13) y actúe como "juez" (però ven faccio sentenzïatore, v. 14) $)^{9}$ La réplica de Piero della Vigna (1.19b) alude desde el incipit Però ch'amore no si pò vedere- a la postura defendida por Iacopo Mostacci (però ch'amore no parse ni pare, v. 8) pero, además, va más allá atacando al autor del soneto y a Giacomo da Lentini en los dos últimos versos del cuarteto: manti ne son di sì folle sapere / che credono ch'amore sia nïente (vv. 3-4). La palabra en rima del tercer verso remite al incipit de Iacopo Mostacci "Solicitanto un poco mio savere" y a la composición del Notaro, perteneciente al debate anterior (1.18b), ed io sì dico che non è neiente (v. 7). Por lo tanto, la polémica se amplía y se pretende rebatir las posturas defendidas en debates anteriores, dentro de la misma escuela ${ }^{10}$. El soneto de Giacomo da Lentini que cierra el ciclo (1.19c) se concentra en la definición del Amor desde el incipit "Amore è uno disio che ven da core"- y en resolver la "duda" planteada en el primer soneto, sin referencias a los contendientes aunque con llamadas internas a las composiciones anteriores.

\subsection{Los sonetos anónimos}

La última disputa del corpus de la escuela siciliana (C) la constituyen dos sonetos anónimos ${ }^{11}$ en los que la discusión gira también en torno a la esencia y naturaleza del Amor. Los dos primeros versos del soneto inicial (25.27) plantean indirectamente una pregunta ("Non truovo chi mi dica chi sia Amore / ove dimori o di che cosa è nato") que el autor dirige a un destinatario no identificado, pero, en realidad, se pretende entrar en un debate para mostrar el error del contendiente (mostrar vi voglio come avete errato v. 8) y rebatir su opinión. Es, en realidad, una pregunta retórica, puesto que el mismo autor ofrece la solución en el verso 9 y la desarrolla a lo largo de la sextina.

El soneto de respuesta (25.28) entra en polémica con un "voi" al que se acusa indirectamente de presunción y prepotencia al negar que esa sea la actitud que él mismo va a adoptar en el debate: "Io no lo dico a voi sentenzïando" (v. 1). El tono irónico es claro a través de este procedimiento antifrástico, puesto que su modo categórico de responder a la pregunta planteada, en los versos

\footnotetext{
${ }^{9}$ M. Picone apunta a Piero della Vigna como destinatario del soneto de Iacopo Mostacci, basándose en su prestigio como autoridad en el campo de las disputationes literarias, "La tenzone de Amore..." p. 23.

${ }^{10}$ El tono violento del ataque viene marcado por el adjetivo folle, utilizado en sentido figurado, con el significado concreto en esta composición: "che muove da passione violenta e inconvenibile; che nasce da assurda e irragionevole convinzione, da stoltezza, da sconsideratezza; che è privo di ragionevole motivazione, insano, disennato (un sentimento, un pensiero, un discorso, uno stato, una condizione, ecc.)". Cfr. S. Battaglia, Grande Dizionario della lingua italiana, UTET, Torino, 1970, t. VI, s.v. folle (16).

${ }^{11}$ Sobre la atribución de la tenzone basada en su colocación en $\mathrm{V}$ y sus afinidades temáticas vid. PSS II, p. 1009.
} 
siguientes, será casi idéntico al del soneto anterior: "Amor nonn-è, se non come cred'eo" v. $9(25.28)^{12}$. El tercer verso alude directamente al debate mediante la expresión "mi parto"13, los verbos de razonamiento y dicción (dicovi, rispondo, dimando ${ }^{14}$ ) y la referencia a la $2^{\mathrm{a}}$ persona:

ca s'eo mi parto con voi ragionando, dicovi parte de lo mio volere, e poi rispondo a lo vostro dimando (PSS, 25.28, vv. 3-5).

Los tres gerundios en rima -sentenzïando (v. 1), ragionando (v. 3), dimando (v. 5)-, que forman parte del exordio, subrayan la justificación de la respuesta que se va a desarrollar en la sextina y la contraargumentación a la tesis anterior.

\section{Marcas técnico-formales}

3.1. Nos hemos propuesto analizar, en estas composiciones de correspondencia en las que, como hemos visto, se debate sobre aspectos de fenomenología amorosa y se contraponen varias opiniones o teorías, cuáles son las técnicas de la argumentación, dentro de la polémica, y cuáles los procedimientos utilizados para articularla desde el punto de vista formal.

En primer lugar, en cuanto a la estructura retórico-argumentativa y, sobre todo, en relación a la terminología técnica, Claudio Giunta ha puesto en evidencia la importancia de la epistolografía y del método escolástico en los géneros poéticos dialogados medievales (Giunta 2002: 194 y ss.). El espíritu dialéctico en el ambiente intelectual medieval va ganando terreno y alcanza plena madurez en el siglo XIII gracias al método didáctico escolástico de la argumentación y demostración, a través del constante ejercicio de plantear problemas, proponer objeciones y réplicas y aportar soluciones. Este esquema es particularmente evidente en el modo racional y lógico de plantear la cuestión a debatir y en el uso de ciertas fórmulas.

La tenzone A, que, como veíamos, se abre con un soneto del Abate di Tivoli, recibe una respuesta de Giacomo da Lentini que concentra en los dos últimos versos de la octava su opinión sobre la cuestión planteada: "ed io sì dico che non è neiente,/ ca più d'un Dio non è né essere osa." (1.18b, vv. 7-8)

La expresión ed io si dico recuerda a la fórmula utilizada en la disputatio escolástica respondeo dicendum como modo de expresar la solución a la cuestión planteada (Grabmann 1980: vol. I, p. 45).

\footnotetext{
${ }^{12}$ En 25.27 tenemos en el v. 4 "Amor nonn-è se non u.nome usato"

13 Antonelli acoge la propuesta de Santangelo que corrige mi parto por mi parllo, con el significado de 'discuto',sobreentendiendo 'disiento', y señala que recuerda al joc partit de los provenzales. Cfr. PSS I, p. 1016,

${ }^{14}$ Con el significado de 'domanda', 'interrogazione', 'richiesta', 'preghiera'. Cfr. GDLI, s.v. domando (dimando).
} 
Los siguientes versos del primer terceto: "E chi lo mi volesse contastare, / io li lo mostreria per quia e quanto" (1.18b, vv. 9-10) también retoman una expresión escolástica, en la que, según Santangelo, el primer término (quia)

indica el procedimento lógico a posteriori, che solo si usava per la dimostrazione dell'esistenza di Dio; la seconda accenna all'argomento della quantità (quanto), a cui si ricorreva quando di Dio si voleva dimostrare l'unità. (Santangelo, apud R. Antonelli, 2008: 368)

Por otro lado, no podemos descartar un cierto tono irónico en el uso de esta expresión, por parte de Giacomo da Lentini en respuesta a L'Abate, puesto que es una fórmula muy específica, empleada para dirimir cuestiones teológicas y, en este caso, el contexto en el que se inserta es bien diverso. Este mismo tono está presente en los últimos versos del soneto continuando con el empleo de un léxico de carácter religioso:

In vanitate non voglio più stare:

voi che trovate novo ditto e canto,

partitevi da ciò, che voi peccate.

(1.18.b, vv. 12-14)

La respuesta del Abate (1.18.c) contiene en el tercer verso el adverbio imprimamente como modo lógico-formal de organizar sus objeciones a la tesis defendida por Giacomo da Lentini.

El primer soneto de la tenzone B, "Solicitando un poco meo savere" $\left(1.19^{\mathrm{a}}\right)$, enviado por Iacopo Mostacci para resolver un "dubio", utiliza la fórmula genérica "On'omo dice", en el primer verso del segundo cuarteto, que se corresponde con el dicitur quod utilizado en las disputas escolásticas. Señala también Antonelli que la expresión ma eo del v. 7, que encabeza la adversativa en la que manifiesta su desacuerdo con la opinión de que el Amor es sustancia, remite al sed contra (PSS I, p 395).

Por otra parte, este soneto recurre a una terminología específica ligada muy directamente al ámbito filosófico y escolástico como los sustantivos dubio (v. 3) o sentenzïatore (v. 14) y los verbos determinare (v. 4) y consentire (v. 7) (PSS I, p. 394 y ss.). El recurso a este vocabulario técnico supone, para Michelangelo Picone, un elemento diferenciador con respecto a los modelos occitánicos (Picone 1979: 23).

Dentro de un léxico más general, son muy frecuentes determinados términos utilizados para articular la argumentación, como los verbos de dicción: saccia dir v. 3, dicono v. 5, (1.18b); parlereste v. 6 (1.18c); dir v. 2, dice v. 7, dirialo v. 11 (1.18d); dire v. 11 (1.19a), chi mi dica v. 1 (25.27); no lo dico v.1, dicovi, v. 4 (25.28); o sustantivos como parlamento v. 6 (1.18d); verbos cognitivos: credo v. 4, credereste v. 7 (1.18c); saccio v. 12 (1.19a); sapere v. 3, credono v. 4, credere v. 12, creduto v. 14 (1.19b); savere v. 2, cred'eo v. 9, pensare v. 12, (25.28). El campo sémico de la polémica se concreta mediante verbos 
que interpelan al interlocutor: contastare ('contestare') v. 9, mostreria v. 10 (1.18b); rispondo v. 5, dimando, v. 5 (25.28) o que remiten directamente al debate: riprende ('rimprovera') v. 11 (1.18c); mi parto ('discuto') v. 3 (25.28) ${ }^{15}$, que se suman a los ya citados más arriba.

Siguiendo con al análisis lingüístico, la estructura explicativa y argumentativa del pensamiento se refleja también mediante la complejidad sintáctica a través de la subordinación y sus correspondientes nexos:

\begin{tabular}{|l|l|}
\hline $\begin{array}{l}1.18 \mathrm{a} \\
\text { v. } 2 \text { ca mi 'ntendiate s'io chero razone: }\end{array}$ & hipotética con valor causal \\
\hline v. 3 cad io son tutto fatto a tua manera, & declarativa causal \\
\hline v. 12 che per mezzo lo core m'ai partuto: & causal \\
\hline $\begin{array}{l}1.18 \mathrm{~b} \\
\text { v. } 3 \text { Per ch'io vi saccia dir lo convenente }\end{array}$ & final \\
\hline v. 8 ca più d'un dio non è né essere osa & causal 'poiché' \\
\hline $\begin{array}{l}1.18 \mathrm{c} \\
\text { v. } 4 \text { ca non credo ca lealmente amiate. }\end{array}$ & con valor causal 'poiché' \\
\hline $\begin{array}{l}\text { v. } 5 \\
\text { coralmente, }\end{array}$ & declarativa causal \\
\hline v. 7 anzi voi credereste veramente & adversativa 'ma' \\
\hline v. 9 Per ciò ch'è di sì scura canoscenza. & completiva \\
\hline $\begin{array}{l}1.19 \mathrm{a} \\
\text { v. } 8 \text { però ch'amore no parse ni pare }\end{array}$ & \\
\hline v. 14 però ven faccio sentenzïatore & valor causal 'poiché' \\
\hline & valor causal 'perciò' \\
\hline $\begin{array}{l}1.19 \mathrm{~b} \\
\text { v. } 1 \text { Però ch'Amore no si pò vedere }\end{array}$ & valor causal 'poiché' \\
\hline v. 5 ma po' ch'Amore si face sentire & adversativa \\
\hline $\begin{array}{l}1.19 \mathrm{c} \\
\text { v. } 7 \text { ma quell'amor che stringe con furore }\end{array}$ & adversativa \\
\hline v. 9 che li occhi rapresentan a lo core & valor declarativo causal \\
\hline 25.27 & \\
v. 3 perché la gente il chiama per segnore & causal \\
\hline $\begin{array}{l}\text { v. } 5-6 \text { Però la gente n'è tutta 'n errore } \\
\text { perch'ogn'omo per lüi è dontato }\end{array}$ & causal \\
\hline v. 7 i. llui nonn-è né forza né valore: & valor concesivo \\
\hline
\end{tabular}

${ }^{15}$ Vid. n. 13. 


\begin{tabular}{|l|l|}
\hline 25.28 & causal \\
v. 3 ca s'eo mi parto con voi ragionando & \\
\hline v. 6 ca 'ntesi che volete voi savere & causal \\
\hline v. 13 ca se deo fosse, non facera reo: & causal \\
\hline v. 14 ca 'n deïtate è tutto degno afare & causal \\
\hline
\end{tabular}

Puede apreciarse en estos casos cómo la construcción hipotáctica, frente a la paratáctica, es mucho más efectiva en el "discurso" argumentativo porque tiene un carácter estratégico que establece relaciones precisas entre los elementos del discurso y por ello guía al lector de una forma muy eficaz; como dice Perelman:

establece unos límites, significa adoptar una posición. Ordena al lector, le obliga a ver ciertas relaciones, reduce las interpretaciones que podría tener en consideración, se inspira en el razonamiento jurídico bien construido. (Perelman-Olbrechts Tyteca 1989: 254)

En el ámbito de la coordinación caba destacar la conjunción ordunque $(1.18 \mathrm{e}, \text { v. } 9)^{16}$, que se sitúa en los tercetos como nexo conclusivo, en el último soneto de la tenzone. También el adverbio ben 'certamente' (1.19c v. 5) tiene un valor afirmativo cuya finalidad es reforzar el argumento. En el caso de imprimamente (1.18c v. 3) su función es la de organizar el discurso.

Conviene, asimismo, hacer alusión al uso argumentativo de pronombres con valor indefinido y de partículas impersonales.

En muchas de las composiciones que hemos acotado llama la atención la presencia de fórmulas impersonales en función de sujeto como: ogn'om (1.18.d, v. 4), on'omo (1.19a, v. 5), om (1.19a, v. 9), ch'om (25.28, v. 10), qual om (1.18c, v. 1$)$, gente $(25.27$, v. 3 y v.5); indefinidos: manti $(1.19 \mathrm{~b}$, v. 3$)$, molti (25.28, v. 11); demostrativos: quelli (1.18.b, v. 4). En todos estos casos el empleo de una tercera persona indefinida con la que se quiere mostrar el desacuerdo obedece a una ambigüedad manifiesta, son fórmulas voluntariamente imprecisas con las que se consigue un efecto de polémica no tan directa, el desacuerdo es más sutil, el ataque al interlocutor contra el que se disiente no es tan directo, es más velado.

3.2. Desde el punto de vista retórico, son varios los recursos empleados para apoyar la argumentación, entre ellos la comparación que, aunque forma parte del ornatus, tiene una gran fuerza probatoria al servicio de la demostración (Lausberg 1966: I, 356).

El soneto de respuesta de l'Abate di Tivoli a Giacomo da Lentini refuerza su opinión, expresada de modo conclusivo en los tercetos, mediante una comparación :

${ }^{16}$ Cr. S. Battaglia GDLI, s.v. ordunque 'dunque', 'quindi'. 
Per ciò ch'è di scura canoscenza, che n'adiven come d'una bataglia: chi stâ veder riprende chi combatte (1.18c, vv. 9-11).

Dentro de la misma tenzone, la respuesta de Giacomo da Lentini al soneto anterior concluye también con una comparación, presente en otros poetas de la escuela y procedente de la lírica provenzal ${ }^{17}$ :

Ma tacciolmi, che no mi sia vergogna, ca d'onn parte amor ò '.pensieri ed entra '. meve com'agua in ispogna. (1.18d, vv. 12-14).

En la tenzone B, Piero della Vigna utiliza como apoyo a su teoría sobre el poder que ejerce el Amor la comparación con el poder de atracción que tiene el imán sobre el hierro ${ }^{18}$ :

Per la vertute de la calamita como lo ferro atra' no si vede, ma sì lo tira signorevolmente; (1.19b, vv. 9-11).

Los versos siguientes que cierran el soneto contienen dos expresiones con valor proverbial ${ }^{19}$ :

Quella ripresa non tegn'e' valenza:

chi accatta 'l mercato, sa che vaglia, chi leva, sente più che quel che batte (1.18c, vv. 12-14).

El uso de expresiones de tono proverbial o sentencioso, como la anterior, o bajo formas analógicas tienen un gran valor argumentativo por su vinculación con la auctoritas y por ello son acuñadas y elevadas a pensamiento de validez general. Consecuentemente, poseen cierto peso para apoyar la opinión mantenida. ${ }^{20}$ Por otra parte, la coincidencia en todos los sonetos de la colocación en los versos finales de estas comparaciones, pertenecientes a la esfera natural y acuñadas previamente por el uso en otros autores, o de las frases de tono sentencio-

\footnotetext{
${ }^{17}$ PSS, I, p. 380, n. 14.

${ }^{18}$ Esta comparación es recurrente en la lírica medieval, desde los provenzales a Petrarca. Vid. PSS I, p. 401, n. 9 .

${ }_{19}$ Con respecto al v. 14, G. Contini apunta: "il verso è un'espressione proverbiale, la quale in origine doveva alludere o allo schiavo o alla bestia che porta un peso e lo sente più di colui che la batte per farglielo portare".Cfr. Poeti del Duecento, Milano-Napoli, Riccardo Ricciardi, 1960, vol. II, t. I, p. 85, n. 14.

20 "En cuanto prueba, la sententia entraña una auctoritas". Cfr. H. Lausberg, Manual..., vol. I, p. 872.
} 
so y proverbial corrobora su función conclusiva y argumentativa. La epidíctica aconsejaba, de hecho, comenzar o concluir un texto con una frase de carácter sentencioso para captar la atención del público o para sintetizar el contenido expuesto (Giunta 2002: 201).

Si los procedimientos anteriores estaban al servicio del desarrollo y conclusión del razonamiento para sostener una opinión o teoría dentro de un debate, también hay ciertas marcas o fórmulas, situadas en el exordio de la composición, que cumplen la función de iniciarlo. Éste es el papel de la interrogación retórica que, en el único caso del corpus, supone una forma hábil de iniciar el razonamiento (Perelman 1989: 255): "Feruto sono isvarïatamente: / Amore m'à feruto, or per che cosa?" (1.18b. vv.1-2).

A la figura anterior se suma el recurso a otros procedimientos como incipit de las composiciones, como por ejemplo, la definición, que tiene la función de precisar los términos de los que se trata en el debate o, como en este caso, de responder a la cuestión planteada desde el inicio dejando clara la postura defendida. Giacomo da Lentini interviene en el debate con Iacopo Mostacci y Piero della Vigna (B) con una definición que cierra la disputa, y que sitúa en el primer cuarteto del soneto, sobre la naturaleza del Amor:

Amor è uno disio che ven da core per abondanza di gran piacimiento, e li occhi imprima generan l'amore e lo core li dà nutricamento.

(1.19c, vv. 1-4).

La tenzone $\mathrm{C}$ propone en el primer soneto la definición, en este caso, en los tercetos. El planteamiento se realiza a través de una interrogación retórica puesto que el mismo autor aporta la solución:

Tre cose sono in una concordanza che tegnono lo corpo i. lor podere, le quali segnoreggiano lo core: piacere e pensare e disïanza; d'este tre cose nasce uno volere, laonde la gente dice che sia Amore. (25.27, vv. 9-14).

También en el incipit de varios sonetos está presente la negación como una de las modalidades de expresión del pensamiento y ésta suele intervenir cuando se argumenta (Perelman 1989: 248). Sin embargo, el número de casos no es suficientemente amplio como para extraer conclusiones más asentadas sobre su empleo $^{21}$.

${ }^{21}$ PSS I: Cotale gioco mai non fue veduto (1.18d); Però ch'Amore no si pò vedere (1.19b); PSS II: Non truovo chi mi dica chi sia Amore (25.27); Io no lo dico a voi sentenzïando (25.28). 


\section{Conclusión}

Las composiciones que hemos analizado, pertenecientes a las primeras tenzoni de la Magna Curia, son textos de polémica y de debate que plantean diversas visiones y teorías sobre el amor, en los que, por tanto, están presentes las distintas técnicas de la argumentación. El alcance de este debate involucra no sólo a los poetas que intervienen en la discusión y a los que casi siempre se cita mediante referencias internas -si exceptuamos la composición 1.18e en la que se menciona directamente a Giacomo da Lentini- puesto que las referencias, mediante diversos procedimientos como los indefinidos o las fórmulas generales, tiene un carácter general y se dirigen a todos aquellos poetas implicados en cuestiones de teoría amorosa. El tono del debate se personaliza y se entra, si no en la descalificación del oponente, sí en la intención de rebatir las propuestas a través de la ironía o el ataque velado. El ataque directo sólo está presente en un caso puntual, en el que Piero della Vigna utiliza el adjetivo folle (1.19b, v. 3) sin mencionar directamente el destinatario pero aludiendo a Giacomo da Lentini, que defiende en la composición anterior una postura contraria a la suya. La ironía impregna la argumentación retórica mediante diversos procedimientos lingüísticos, como el uso de un léxico muy específico, las fórmulas impersonales, el uso de los indefinidos, etc. que, elevando a categoría general la discusión, suavizan la confrontación y eluden el ataque directo.

\section{Bibliografía}

\subsection{Abreviaturas bibliográficas}

GDLI: Grande Dizionario della lingua italiana (dir. Salvatore Battaglia), Torino, UTET, 1970.

PSS: I poeti della Scuola Siciliana, Edizione promossa dal centro di studi filologici e linguistici siciliani, Milano, Mondadori, 2008; vol. I: Roberto Antonelli (ed.), Giacomo da Lentini; vol. II: Costanzo di Girolamo (dir. ), Poeti della corte di Federico II.

\subsection{Referencias bibliográficas}

ANTONELLI, Roberto (ed.) (2008): Giacomo da Lentini, Centro di Studi Filologici e Linguistici Siciliani, Milano, I Meridiani Mondadori, vol. I.

BATTAGLIA, Salvatore (dir.) (1970): Grande Dizionario della lingua italiana, Torino, UTET.

BOOTH WAYNE C. (1989): Retórica de la ironía (versión cast. Jesús Fernández Zulaica y Aurelio Martínez Benito), Madrid, Taurus.

CIAN, Vittorio (1923): Storia dei generi letterari italiani. La satira, Milano, Vallardi. 
CONTINI, Gianfranco (ed.) (1960): Poeti del Duecento, Milano, Riccardo Ricciardi.

DI GIROLAMO, Costanzo (ed.) (2008): Poeti della corte di Federico II, Centro di Studi Filologici e Linguistici Siciliani, Milano,I Meridiani, Mondadori, vol. II.

GIUNTA, Claudio (2002): Versi a un destinatario. Saggio sulla poesia italiana del Medioevo, Bologna, Il Mulino.

- (2002): Due saggi sulla tenzone, Roma, Antenore.

GRABMANN, Martin (1980): Storia del metodo scolastico, Firenze, La Nuova Italia.

LAUSBERG, Henrich (1966): La retórica literaria. Fundamentos de una ciencia de la literatura, (trad. J. Pérez Riesco), Madrid, Gredos, 3 vols.

MARCENARO, Simone (2010): "Polemiche letterarie nella lirica italiana del Duecento" Revista de Filologia Románica, 27: 77-99.

MOLINIER, Guilhem (1977): Las Flors del Gay Saber estier dichas Las Leys d'Amors, (reimpr. ed. 1841-1843), Genève, Slatkine.

PERELMAN, Chaïm - OLBRECHTS-TYTECA, Lucie (1989): Tratado de la argumentación. La nueva retórica, (J. Sevilla trad.), Madrid, Gredos.

PICONE, Michelangelo (1979): "La tenzone de Amore fra Iacopo Mostacci, Pier della Vigna e il notaio" Il genere "tenzone" nelle letterature romanze delle origini, a cura di Matteo Pedroni e Antonio Staüble, Ravenna, Longo editore.

SANTANGELO, Salvatore (1928): Le tenzoni poetiche nella letteratura italiana delle origini, Genève, Olschki. 